\title{
Reversible band-gap engineering in carbon nanotubes by radial deformation
}

\author{
O. Gülseren, ${ }^{1,2}$ T. Yildirim, ${ }^{1}$ S. Ciraci, ${ }^{3}$ and Ç. Kılıç,* \\ ${ }^{1}$ NIST Center for Neutron Research, National Institute of Standards and Technology, Gaithersburg, Maryland 20899 \\ ${ }^{2}$ Department of Materials Science and Engineering, University of Pennsylvania, Philadelphia, Pennsylvania 19104 \\ ${ }^{3}$ Department of Physics, Bilkent University, Ankara 06533, Turkey
}

(Received 18 April 2001; revised manuscript received 11 December 2001; published 28 March 2002)

\begin{abstract}
We present a systematic analysis of the effect of radial deformation on the atomic and electronic structure of zigzag and armchair single wall carbon nanotubes using the first-principle plane wave method. The nanotubes were deformed by applying a radial strain, which distorts the circular cross section to an elliptical one. The atomic structure of the nanotubes under this strain are fully optimized, and the electronic structure is calculated self-consistently to determine the response of individual bands to the radial deformation. The band gap of the insulating tube is closed and eventually an insulator-metal transition sets in by the radial strain which is in the elastic range. Using this property a multiple quantum well structure with tunable and reversible electronic structure is formed on an individual nanotube and its band lineup is determined from first principles. The elastic energy due to the radial deformation and elastic constants are calculated and compared with classical theories.
\end{abstract}

DOI: 10.1103/PhysRevB.65.155410

PACS number(s): 73.22. $-\mathrm{f}, 62.25 .+\mathrm{g}, 61.48 .+\mathrm{c}, 71.30 .+\mathrm{h}$

\section{INTRODUCTION}

Modification of electronic properties of condensed systems by an applied external pressure or strain in the elastic range have been subject of active study. However, in most of the cases, the changes one can induce by the elastic deformation is minute even negligible due to the rigidity of the crystals. On the other hand, the situation is rather different for single wall carbon nanotubes (SWNT's) owing to their tubular geometry. ${ }^{1-9}$ SWNT's are highly flexible and have a very large Young's modulus. They sustain remarkable elastic deformations, and it has been shown that the structure and electronic properties undergo dramatic changes by these deformations. ${ }^{10-20}$ Similarly, significant radial deformation of SWNT's can be realized in the elastic range, whereby the curvature is locally changed. This way, zones with higher and lower curvatures relative to the undeformed SWNT can be attained on the same circumference. Hence, one expects that radial deformation can induce important modifications in the electronic and conduction properties of nanotubes. ${ }^{21-27}$

Tight-binding calculations have indicated that a SWNT may undergo an insulator-metal transition under a uniaxial or torsional strain. ${ }^{18,19}$ Multiprobe transport experiments ${ }^{20}$ on individual SWNT's showed that the electronic structure can be modified by bending the tube, or by applying a circumferential deformation. Empirical extended Hückel calculations ${ }^{10}$ predicted that the conductance of an armchair SWNT can be affected by the circumferential deformations and a band gap can develop on a metallic armchair SWNT upon twisting. The effect of the radial deformation and squeezing have been investigated by using various methods. $^{21-27}$ However, in spite of these theoretical studies, ${ }^{24,25}$ a systematic analysis of the effect of the radial deformation on the electrical properties has not been carried out yet.

The objective of this paper is to provide a better understanding of the effect of radial deformation on the electronic band structure and elastic properties of SWNT's, based on the extensive first-principle (ab initio) total energy and electronic structure calculations with fully optimized structures. In the next section, a brief review of the the first-principle pseudopotential plane wave method that we used will be given. The effect of the radial deformation on the atomic structure will be discussed in Sec. III A. We discuss the elastic properties of SWNT's under radial strain in Sec. III B. We show that the calculated elastic deformation energies as a function of radial strain can be described very well within the classical theory of elasticity. In Sec. III C, we discuss the effect of the radial deformation on the electronic properties. We find that zigzag nanotubes are metallized under radial deformation in the elastic range. In Sec. III D, this property is exploited to realize various quantum well structures on a single nanotube with tunable electronic properties. We applied two different radial deformations to two adjacent regions of a $(8,0)$ nanotube to generate band offsets at the interface, which in turn lead to multiple quantum well structures. Our conclusions are given in Sec. IV.

\section{METHODOLOGY}

The first-principles total energy and electronic structure calculations have been performed using the pseudopotential plane wave method ${ }^{28}$ within the generalized gradient approximation (GGA) ${ }^{29}$ Calculations have been carried out within periodically repeating supercell geometry because of the necessity of using the periodic boundary conditions with the plane wave method. We used a tetragonal supercell with lattice constants $a_{\mathrm{sc}}, b_{\mathrm{sc}}$, and $c_{\mathrm{sc}}$. The lattice constants $a_{\mathrm{sc}}$ and $b_{\mathrm{sc}}$ are chosen such that the interaction between nearest neighbor tubes is negligible (the minimum $\mathrm{C}-\mathrm{C}$ distance between two nearest neighbor tubes is taken as $6.2 \AA$ ). The lattice constant along the axis of the tube $c_{\mathrm{sc}}$ is taken to be equal to the one-dimensional (1D) lattice parameter $c$ of the tube. The tube axis is taken along the $z$ direction, and the circular cross section lies in the $(x, y)$ plane. In the 1D Bril- 
louin zone (BZ), the wave vector $k_{z}$ varies only along the $z$ axis.

Plane waves up to an energy cutoff of $500 \mathrm{eV}$ are used. With this energy cutoff and using ultrasoft pseudopotentials for carbon atoms, ${ }^{30}$ the total energy converges within 0.5 meV/atom. In addition to this, finite basis set corrections ${ }^{31}$ are also included. Owing to the very large lattice constants of the supercell $a_{\mathrm{sc}}$ and $b_{\mathrm{sc}}$, k-point sampling is done only along the tube axis. The Monkhorst-Pack special k-point scheme $^{32}$ with with $0.02 \AA^{-1} k$-point spacing resulting 5 and $10 \mathbf{k}$ points within the irreducible BZ of the tetragonal supercell are used for $(n, 0)$ and $(n, n)$ tubes, respectively.

\section{RADIALLY DEFORMED NANOTUBES}

\section{A. Geometric structure}

The radial deformation that is treated in this study is generated by applying uniaxial compressive stress $\sigma_{y y}$ on a narrow strip on the surface of a SWNT. In practice such a deformation can be realized by pressing the tube between two rigid and flat surfaces. As a result, the radius is squeezed in the $y$ direction, while it is elongated along the $x$ direction, and hence the circular cross section is distorted to the elliptical one with major and minor axis $a$ and $b$, respectively. A natural variable to describe the radial deformation is the magnitude of the applied strain along the two axes

$$
\epsilon_{y y}=\frac{R_{0}-b}{R_{0}}
$$

and

$$
\epsilon_{x x}=\frac{R_{0}-a}{R_{0}},
$$

where $R_{0}$ is the radius of the undeformed (zero strain) nanotube. We note that the point group of the undeformed nanotubes is $D_{n h}$ or $D_{n d}$ for $n$ even or odd, respectively. Under radial deformation described above, the point group becomes $C_{2 h}$ or $D_{2 h}$ (see Fig. 1). However, depending on the nanotube orientation around the tube axes the in-plane mirror symmetry can be broken. For the $(6,6)$ tube, we studied several different orientations in order to investigate the effect of mirror symmetry on the band crossing at the Fermi level. Three different orientations with point groups $C_{2 v}, C_{2}$, and $D_{2}$ are shown in Fig. 1(d).

For different values of strain $\epsilon_{y y}$, we carried out full structural optimization under the constraint that the minor axis was kept fixed at a preset value. The strains are in the elastic range, since the deformed tubes relax back to the undeformed state when the applied strain is removed. The structural relaxation is done in following steps: first, depending on rotational orientation of the SWNT, either a single bond or a carbon atom at both ends of the minor axis are pressed towards each other by $\left(1-\epsilon_{y y}\right) R_{0}$ and are kept fixed. Then, under this constraint, the coordinates of the remaining atoms and the lattice parameter of the tube $c$ are optimized. At this step, some resultant forces remain on the fixed atom(s) with components opposite to the applied strain a) $(7 \times 0)$
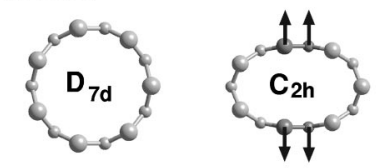

b) $(8 \times 0)$

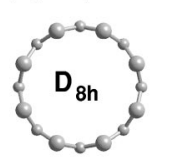

c) $(9 \times 0)$

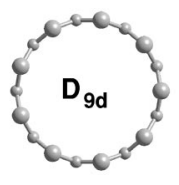

d) $(6 \times 6)$
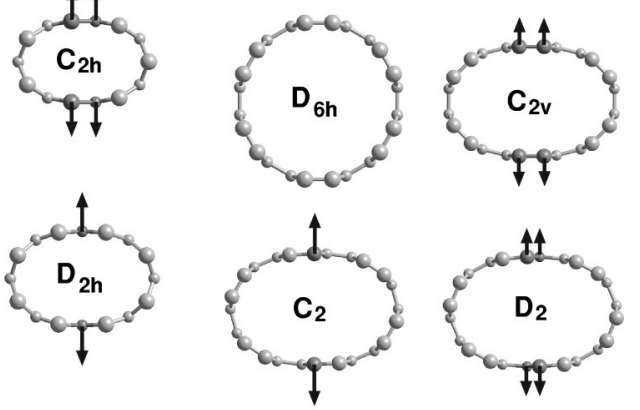

FIG. 1. Top view of the undeformed and deformed nanotubes. The arrows indicate restoring forces on the fixed carbon atoms. The in-plane mirror symmetry can be broken depending on the rotational orientation of the tube [see (d)]. The corresponding point groups of nanotubes are also indicated.

as well as perpendicular to it. In the second step, the fixed bond lengths are optimized together with all the internal coordinates of the atoms and $c$ parameter. Eventually, in the final fully relaxed structure the only remaining force is the restoring force, opposite to the applied strain, on the fixed atoms. All other force components on these fixed carbon atoms and all the forces on the rest of carbon atoms are optimized to be less than $0.01 \mathrm{eV} / \AA$. Figure 1 shows the crosssectional view perpendicular to the tube axis of the fully optimized undistorted and distorted SWNT's as well as the restoring force vector.

Figure 2(a) shows the pair distribution function in a deformed and undeformed $(7,0)$ SWNT. The first peak in Fig. 2(a) corresponds to the first nearest neighbor distance, which is slightly broadened without a shift of the peak position with deformation. This indicates that the $\mathrm{C}-\mathrm{C}$ bond lengths $(\approx 1.41 \AA)$ are practically unaltered under the applied strain. Similarly, the second peak in Fig. 2(a) is also slightly broadened, indicating a small effect of the distortion on the second nearest neighbor distances. The effect of the radial deformation becomes apparent only for the third and further nearest neigbor distances.

Given that first and second nearest neighbor distances did not change significantly with the radial deformation, the only remaining degrees of freedom is the bond angle as clearly seen from the angular distribution function shown in Fig. 2(b). The main peak around $120^{\circ}$ does not change with applied strain, but the other peak a few degrees below the main peak for undeformed tube splits into $j$ new peaks where $j$ is the number of peaks in the radius distribution of zigzag $(7,0),(8,0)$, and $(9,0)$ SWNT's. On the other hand, for the armchair $(6,6)$ SWNT, although the main peak is not changed with strain, the second peak is broadened by a few degrees. One direct consequences of this observation is that for zigzag tubes the lattice parameter $c$ decreases very slightly with radial strain, whereas it is almost constant for the $(6,6)$ SWNT. 

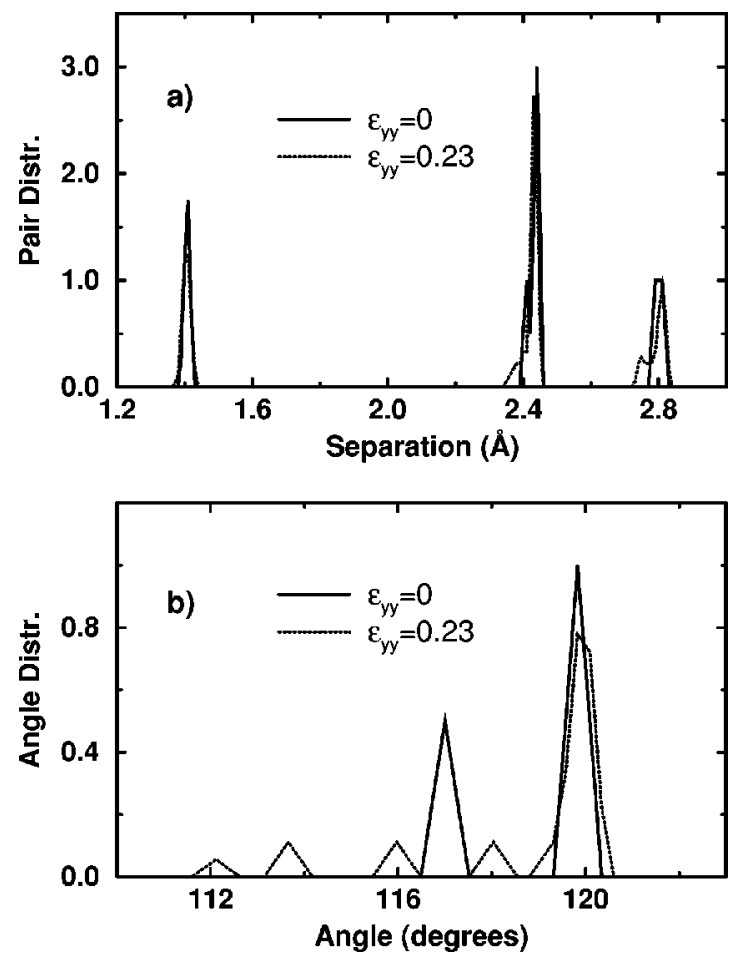

FIG. 2. (a) Pair distribution; (b) bond angle distribution functions for the $(7,0)$ SWNT. Solid line is for undeformed SWNT while the dotted line is for radially deformed one.

In summary, the radial deformation does not have a noticeable effect on the first and second nearest neighbor C-C distances but it induces significant changes in the bond angles. This observation is therefore important and has to be taken into account in tight binding studies of SWNT's with radial deformation.

\section{B. Elasticity}

In order to describe the in-plane elasticity and deformation of the SWNT's, we use first-principle calculations of the elastic deformation energy, i.e., the amount of energy stored in a SWNT as a result of radial deformation, and the classical theory of elasticity. The relation between stress and strain is given by generalized Hooke's law, for the radial deformation described in the previous section

$$
\sigma_{x x}=0=C_{11} \epsilon_{x x}+C_{12} \epsilon_{y y}
$$

and

$$
\sigma_{y y}=\frac{F_{y}}{A}=C_{12} \epsilon_{x x}+C_{11} \epsilon_{y y},
$$

where $F_{y}$ is the restoring force applied on the surface area $A$. $C_{11}$ and $C_{12}$ are the in-plane elastic stiffness constants. Assuming the validity of the Hooke's law, the strain energy becomes a quadratic function of strain as

$E_{T}\left(\epsilon_{x x}, \epsilon_{y y}\right)=E_{T}(0)+\frac{1}{2} \Omega\left(C_{11} \epsilon_{x x}^{2}+C_{11} \epsilon_{y y}^{2}+2 C_{12} \epsilon_{x x} \epsilon_{y y}\right)$.

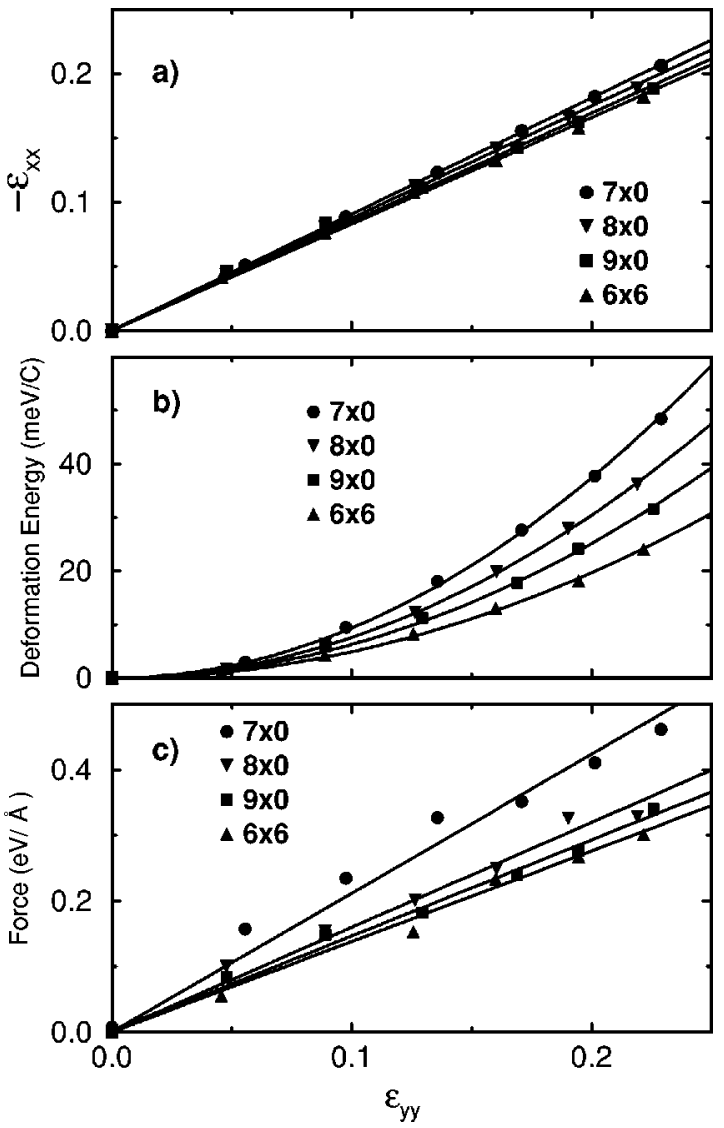

FIG. 3. (a) The strain component $\epsilon_{x x}=\left(R_{0}-a\right) / R_{0}$ along the major axis as a function of applied strain $\epsilon_{y y}=\left(R_{0}-b\right) / R_{0}$. The slope is the in-plane Poisson ratio $\nu_{\|}$. (b) Variation of the elastic deformation energy per carbon atom. (c) The restoring force on fixed carbon atoms. For $(8,0)$ SWNT, the force is scaled by 0.5 since it is only on one carbon atom, while for the other tubes it is on two carbon atoms.

The in-plane Poisson's ratio, $\nu_{\|}$, relates $\epsilon_{x x}$ and $\epsilon_{y y}$, from Eq. (3)

$$
\nu_{\|}=-\frac{\epsilon_{x x}}{\epsilon_{y y}}=\frac{C_{12}}{C_{11}} .
$$

The strain components are plotted in Fig. 3(a). As presented in Table I, $\nu_{\|}$decreases with increasing nanotube radius and is slightly smaller than 1.0. Equations (4) and (5) can be cast in a simpler form by introducing $\nu_{\|}$:

TABLE I. In-plane elastic constants of SWNT's. All elastic constants are in GPa except $\nu_{\|}$which is unitless. $C_{\text {eff }}=C_{11}\left(1-\nu_{\|}^{2}\right)$.

\begin{tabular}{cccccc}
\hline \hline & Radius $(\AA)$ & $\nu_{\|}$ & $C_{\text {eff }}$ & $C_{11}$ & $C_{12}$ \\
\hline$(7,0)$ & 2.76 & 0.904 & 129.88 & 713.36 & 645.15 \\
$(8,0)$ & 3.14 & 0.874 & 98.70 & 416.88 & 364.20 \\
$(9,0)$ & 3.52 & 0.864 & 91.02 & 319.67 & 270.36 \\
$(6,6)$ & 4.06 & 0.828 & 86.12 & 273.46 & 226.34 \\
\hline \hline
\end{tabular}




$$
\sigma_{y y}=\frac{F_{y}}{A}=C_{11}\left(1-\nu_{\|}^{2}\right) \epsilon_{y y}=C_{\text {eff }} \epsilon_{y y}
$$

and

$$
E_{D}=\Omega\left[\frac{1}{2}\left(1+\nu_{\|}^{2}\right) C_{11}-\nu_{\|} C_{12}\right] \epsilon_{y y}^{2},
$$

where $E_{D}$ is the elastic deformation energy obtained from the difference between the total energies of radially deformed and undeformed SWNT's expressed in Eq. (5) [i.e., $\left.E_{T}\left(\epsilon_{x x}, \epsilon_{y y}\right)-E_{T}(0)\right]$. At this point we examine how the stress and the elastic deformation energy calculated from first principles compare with the linear and quadratic forms in Eqs. (7) and (8) obtained from classical theory. To this end, we plot $E_{D}$ and the corresponding restoring forces $F_{y}$ as a function of $\epsilon_{y y}$ in Figs. 3(b) and 3(c), respectively. Interestingly, the quadratic form obtained from classical theory fits very well to the elastic deformation energy calculated from the first principles. Hooke's relation, and hence elastic character of the deformations, persists up to $\epsilon_{y y}=0.25$. It is also noted that the SWNT becomes stiffer as $R$ decreases. The variation of the restoring forces is expected to be linear in the elastic range. The restoring forces in Fig. 3(c) are in overall agreement with this argument, except the deviations at certain data points due to uncertainties in the first-principle calculations, which are amplified because the force is a derivative quantity. Calculated elastic constants are listed in Table I. It is interesting to note that there are discrepancies in the theoretical results for Young's modulus, due to the assignment of the thickness $h$ of the tube wall. ${ }^{33,34}$ Two commonly used values are $3.4 \AA$ (based on graphite interlayer spacing) and $0.6 \AA$ (based on the $\pi$ orbital extent). The wall thickness $h$ can be estimated from the present radial deformation data first by calculating the volume, $\Omega$ from Eq. (8). Then, $h$ is solved by assuming that the tube is a slab with thickness $h$. From this analysis, we found that $h$ is radius dependent and it decreases from $0.88 \AA$ for $(7,0)$ tube to $0.74 \AA$ for $(6,6)$ tube.

\section{Electronic structure}

We now discuss in detail the electronic structure of SWNT's under applied radial strain. The calculated band structures of undeformed and radially deformed zigzag $(7,0),(8,0),(9,0)$ and armchair $(6,6)$ SWNT's are presented near the Fermi level in Fig. 4. The band gaps of zigzag tubes reduce with applied strain, and eventually vanish leading to metallization. Figure 5 summarizes the variation of band gap and density of states at the Fermi level $\mathcal{D}\left(E_{F}\right)$ as a function of the applied strain. For $(7,0)$ and $(8,0)$ SWNT's the band gaps decrease monotonically and the onset of an insulatormetal transition follows with the band closures occurring at different values of strain. Upon metallization $\mathcal{D}\left(E_{F}\right)$ increases with increasing strain. The behavior of the $(9,0)$ tube is, however, different. Initially, the band gap increases with increasing strain, but then decreases with strain exceeding a certain threshold value and eventually diminishes. For all these zigzag SWNT's the band gap strongly depend on the magnitude of the deformation, and $E_{g}$ is closed at 13,22 , and $17 \%$ strain for $(7,0),(8,0)$, and $(9,0)$ nanotubes, respectively.
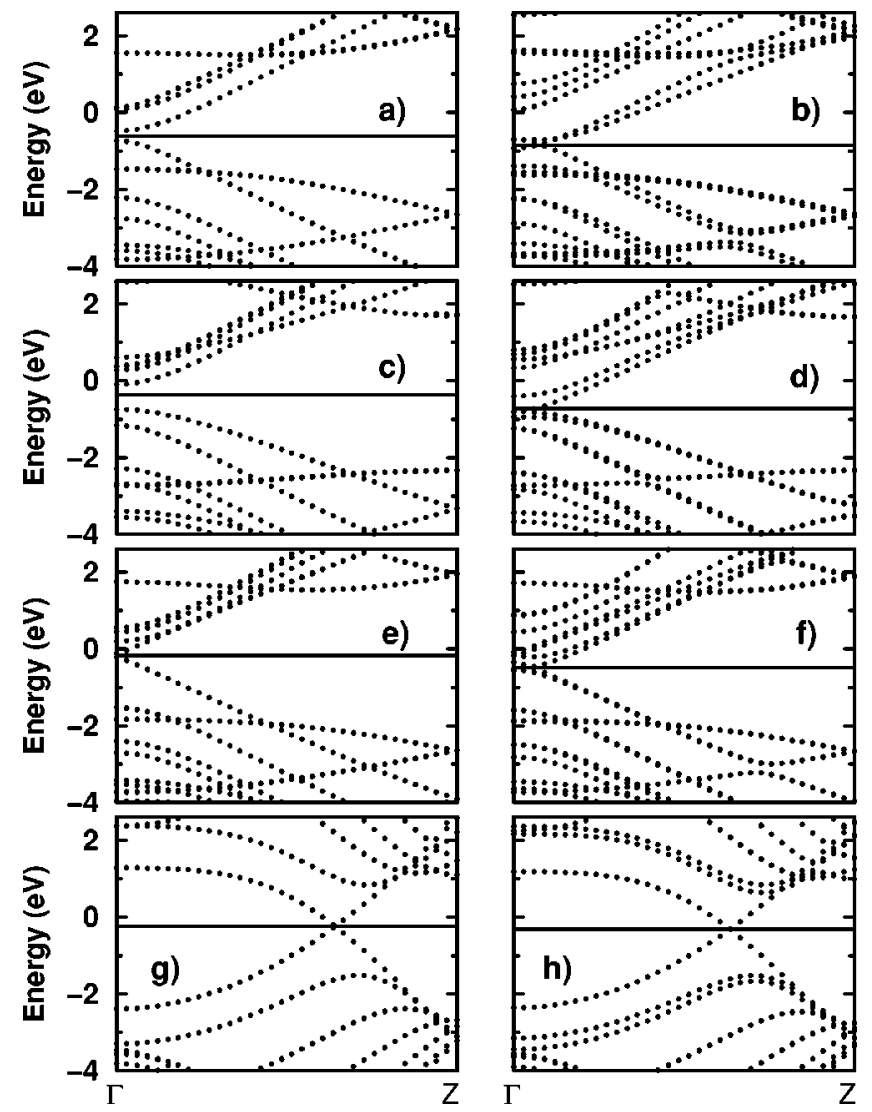

FIG. 4. Energy band structures of undeformed (left) and radially deformed $\left(\epsilon_{y y}=0.23\right)$ (right) SWNTs along the $\Gamma-Z$ direction: (a) and (b) $(7,0)$, (c) and (d) $(8,0)$, (e) and (f) $(9,0)$, (g) and (h) $(6,6)$. Solid line is the Fermi level.

Whereas, the armchair $(6,6)$ SWNT, which is normally metallic, remains metallic with a slowly decreasing $\mathcal{D}\left(E_{F}\right)$ even for significant radial deformation. Earlier Delaney et al. ${ }^{35,36}$ showed that the $\pi^{*}$-conduction and $\pi$-valence bands of a $(10,10)$ tube which normally cross at the Fermi level with quasilinear dispersion, open a pseudogap in the range of $\sim 0.1 \mathrm{eV}$ at certain directions of the $\mathrm{BZ}$ perpendicular to the axis of the tube owing to tube-tube interactions in a rope. The opening of the gap is caused by the broken mirror symmetry. Lammert et al. ${ }^{15}$ pointed out the gapping by squashing $(20,20)$ and $(36,0)$ metallic tubes, since circumferential regions are brought into close proximity. Uniaxial stress of a few kilobars can reversibly collapse a small radius tube inducing a $0.1 \mathrm{eV}$ gap, while the collapsed large radius tubes are stable. In the study of Park et al., ${ }^{24}$ the bandgap of the $(5,5)$ tube were monotonically increasing probably due to bilayer interactions, since the separation of the two nearest wall of the tube became comparable to the interlayer distance of graphite.

In order to explain the band gap variation of $(n, 0)$ tubes, the energies of a few bands near the band gap are plotted as a function of strain in Fig. 6. The singlet $\pi^{*}$ state in the conduction band shifts downwards in energy much faster than the other states do with increasing strain. This is due to the increasing curvature with increasing radial deformation. Since the singlet $\pi^{*}$ state lies below the double degenerate 

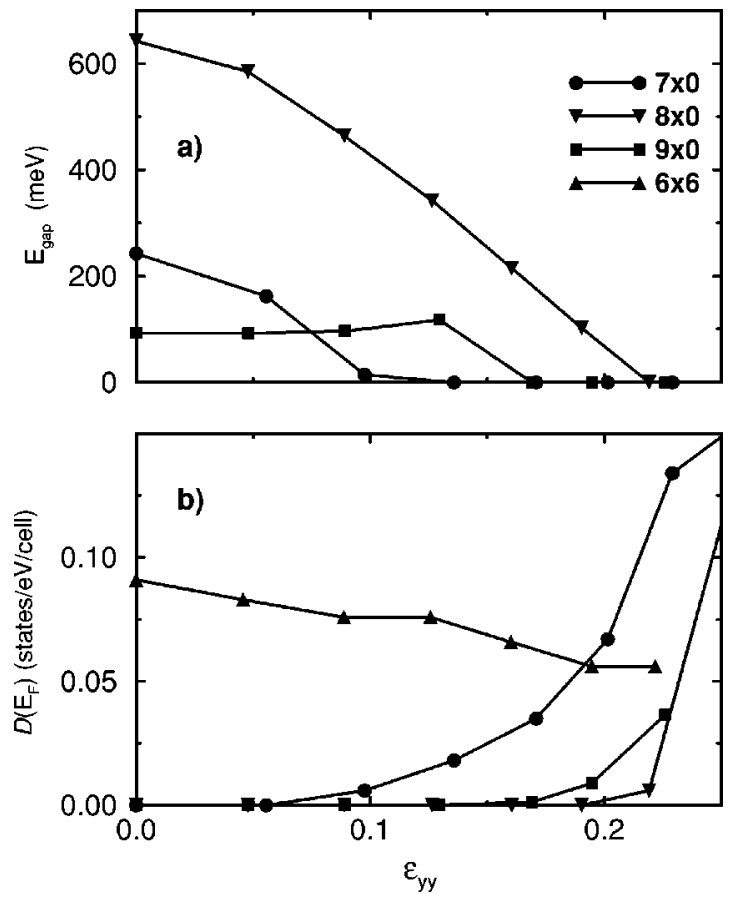

FIG. 5. The variation of the band gap $E_{g}$ (a) and the density of states at the Fermi level $\mathcal{D}\left(E_{F}\right)$ (b) as a function of applied strain $\epsilon_{y y}$.

$\pi^{*}$ states for both $(7,0)$ and $(8,0)$ SWNT's, their band gaps are closed monotonically with increasing $\epsilon_{y y}$. On the other hand, for the $(9,0)$ SWNT this singlet $\pi^{*}$ state is above the double degenerate $\pi^{*}$ states. The increase of the band gap at the initial stages of radial deformation is connected with relatively higher rate of downward shift of the double degenerate $\pi$-valence band relative to the $\pi^{*}$-conduction band under low strains. Once the singlet $\pi^{*}$ band, which shows faster decrease with strain, crosses the doublet conduction band and enters into the gap, the band gap begins to decrease with increasing strain.

Finally, we examined the effect of the radial deformation on the charge density. In Fig. 7 we show the charge density
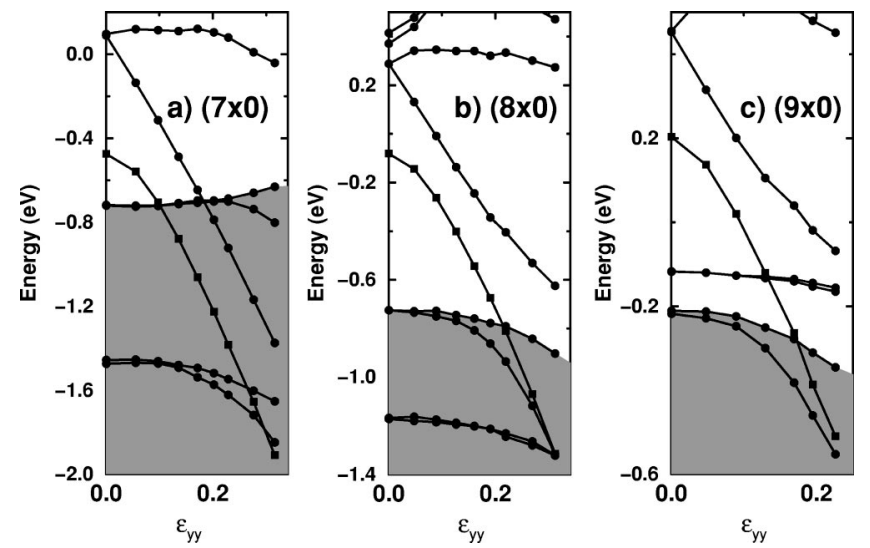

FIG. 6. The variation of energy eigenvalues of states near the band gap at the $\Gamma$ point of the $\mathrm{BZ}$ as a function of the applied strain. The shaded region is the valance band. The singlet state originating in the conduction band is indicated by squares.

\section{$(9,0)$ SWNT}

$$
\varepsilon_{y y}=0
$$
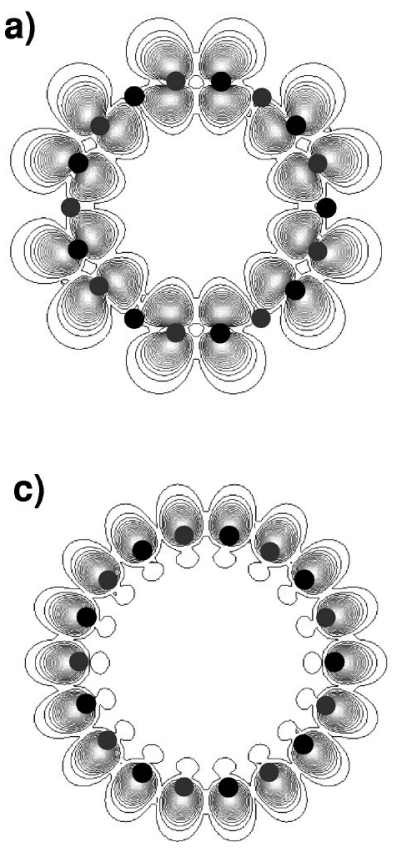

$\varepsilon_{\text {yy }}=0.23$

b)

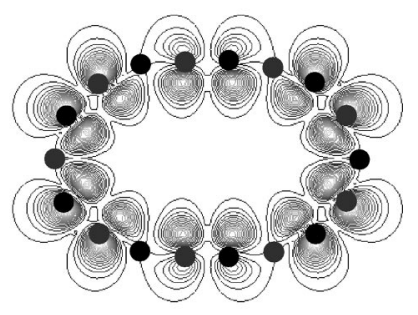

d)

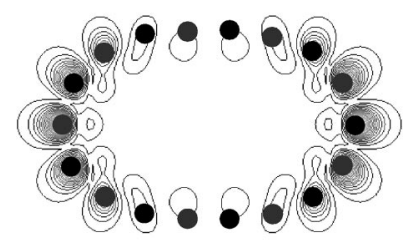

FIG. 7. Charge density of the highest valence band state at the $\Gamma$ point of a undeformed (a) and deformed (b) $(9,0)$ SWNT. The panels (c) and (d) show the the singlet "conduction" band states.

of states near the band edges. The effect of the deformation is remarkable on the singlet state; charge moves from the low curvature regions to the high curvature regions as the strain is increased. Significant charge rearrangements with radial deformation can modify the chemical activity of the surface of the SWNT relative to foreign atoms and molecules. Since a SWNT can sustain large elastic deformations, it allows significant charge rearrangements on its surface. Hence, this effect can be used to control chemical reactivity of specific carbon atoms in SWNT's. ${ }^{37}$

\section{Strain induced quantum structures}

It is clear from the above discussion that the band gap of an insulating SWNT can be modified, and even an insulatormetal transition can be induced by radial deformation in the elastic range. If the applied deformation is not uniform but has different strength at different zones of the tube, it renders variable electronic structure along the tube axis. For example, each zone of an individual SWNT undergoing different radial deformation attains a different band gap. Owing to the band offsets at the junction, quantum structures can be engineered on an individual tube.

Experimental and theoretical methods have been proposed in the past to determine the band offsets, and hence to reveal the band diagram perpetuating along the superlattice axis. ${ }^{38}$ However, there are some ambiguities in the case of SWNT superlattices $\left(A_{n} B_{m}\right)$, which are formed by periodically re- 
peating undeformed $A$ regions (of $n$ unit cells) and radially deformed $B$ regions (of $m$ unit cells): (i) The alignment of the valence and conduction bands of the $A$ and $B$ regions and the resulting band lineup is a complex process involving charge transfer between $A$ and $B$, and also modification of the crystal potential in the deformed region. In fact, the above analysis of radial deformation has shown that the valence and conduction band edges can be lowered when a zigzag SWNT is radially deformed. Therefore, a realistic treatment of the band alignment requires self-consistent calculation of the crystal potential. (ii) Even if the band diagram were known, it is not obvious whether the effective mass approximation (EMA) is applicable for an individual, nonuniformly deformed SWNT. Therefore, instead of applying EMA to a 1D real space band diagram or quantum well structure, one has to perform electronic structure calculations on the $\left(A_{n} B_{m}\right)$ supercell. However, $a b$ initio calculations become tedious for large supercell size owing to many involved carbon atoms. Earlier, we used a tight binding method and showed that states at the band edges are confined in either $A$ or $B$ regions of a superlattices $\left(A_{8} B_{8}\right),\left(A_{4} B_{12}\right)$ and $\left(A_{12} B_{4}\right)$ formed on an individual $(7,0)$ SWNT. $^{26}$

In this study, we extend our earlier calculations of quantum structures on SWNT's and present an ab initio analysis of band lineup of the $\left(A_{6} B_{6}\right)$ superlattice formed on an individual $(8,0)$ SWNT. Similar to the previous model, here the $A$ region is left undeformed, but the $B$ region is radially deformed by $\epsilon_{y y}=0.16$. This system consists of 384 carbon atoms in a supercell involving 12 original unit cells of the $(8,0)$ SWNT. We performed a partial structural optimization for the $\left(A_{6} B_{6}\right)$ superlattice, since the full optimization is not tractable within a reasonable computation time. We first optimized single unit cells in the $A$ and $B$ regions corresponding to $\epsilon_{y y}=0$ and $\epsilon_{y y}=0.16$, respectively. Then, we connected $A$ and $B$ regions smoothly by one intermediate unit cell. Finally, fully self-consistent electronic band structure calculations were carried out on this structure. In Fig. 8 we show the planar $(x y)$ averaged self-consistent potential $\bar{V}_{c}(z)=\int_{S} V_{c}(\mathbf{r}) d x d y / S$. Here $S$ is the $x y$ cross section of the supercell. The alignment of the valence band edges between undeformed $A$ and deformed $B$ are revealed by first integrating the planarly averaged potential $\bar{V}_{X}=\int_{l} \bar{V}_{c}(z) d z / l$ at each region $(X=A$ or $B$ ) over a length of original unit cell (i.e., $l=c$ ) along the tube axis. ${ }^{39}$ In Fig. $8, \bar{V}_{X}$ is shown for both regions of the supercell which constitutes the reference level for the band lineup. In the next step, we determine the energy of the valence band edge from the $\bar{V}_{A}^{\infty}$ and $\bar{V}_{B}^{\infty}$ calculated for two different, uniform (infinite) $(8,0)$ SWNT's (one undeformed, the other uniformly deformed with $\epsilon_{y y}=0.16$ ). These are $E_{V, A}^{\infty}$ and $E_{V, B}^{\infty}$. It is assumed that in the nanotube superlattice $E_{V, A}^{\infty}$ and $E_{V, B}^{\infty}$ are unaltered. The band lineup of the valence band is calculated from the difference $\Delta E_{V}$ $=\bar{V}_{A}-\bar{V}_{B}+E_{V, A}^{\infty}-E_{V, B}^{\infty}$. For $(8,0)$ SWNT, we find $\Delta E_{V}$ $\sim 180 \mathrm{meV}$; the valence band edge of $B$ is lower than that of

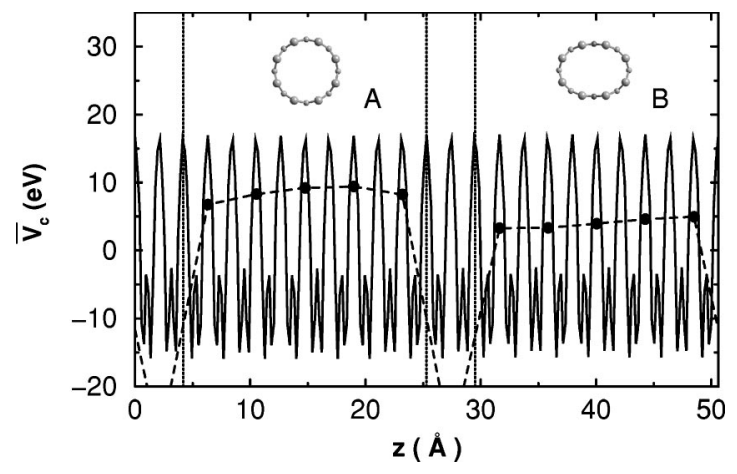

FIG. 8. The planar averaged crystal potential along the axis of the $A_{6} B_{6}$ superlattice nanotube. Dotted vertical lines show the interfaces. Circular cross section of the undeformed $(8,0)$ nanotube in region $A$ and elliptical cross section of radially deformed nanotube $\left(\epsilon_{y y}=0.16\right)$ in region $B$ are shown in the inset. The potential averaged over the original unit-cells of nanotube is shown by circles and dashed line. This average potential scaled by 25 for clarity.

$A$ indicating a staggered band lineup. This result clearly shows that by applying periodic radial deformation on an individual semiconducting SWNT one can generate a quantum structure, where the band gap in the direct space along the tube axis undergoes a periodic variation which is continuously tunable and reversible.

\section{CONCLUSIONS}

In this work we present an extensive first-principle analysis of the effect of radial deformation on the atomic structure, energetics and electronic structure of SWNT's. We find that the energy band structure and the variation of the gap with radius (or $n$ ) differs from what one derived from the zone folded band structure of graphene based on simple tight binding calculations. More interestingly, the response of the energy bands around the band gap to the applied radial deformation is different for different bands. Depending on the relative position of these bands, the band gap displays different behavior under the radial deformation. In general, the band gap is reduced and eventually closed to yield an insulator-metal transition under the elastic radial deformation. The strong dependence of the band gap on the applied strain, its reversible and continuously tunable behavior are exploited to form quantum well structure on an individual SWNT. A first-principle calculation of the alignment of the valence band is presented. The deformation energy and elastic constants under the radial deformation are calculated. We find that the strain energy due to the radial deformation can be fitted very well to the quadratic expressions obtained from the classical theory of elasticity within the Hooke's law.

\section{ACKNOWLEDGMENTS}

This work was partially supported by the National Science, Foundation under Grant Nos. INT01-15021 and TÜBÍTAK under Grant No. TBAG-U/13(101T010). 
*Present address: National Renewable Energy Laboratory, Golden, CO 80401.

${ }^{1}$ S. Iijima, Nature (London) 354, 56 (1991).

${ }^{2}$ M.S. Dresselhaus, G. Dresselhaus, and P.C. Eklund, Science of Fullerenes and Carbon Nanotubes (Academic Press, San Diego, 1996).

${ }^{3}$ M.S. Dresselhaus, G. Dresselhaus, and R. Saito, Phys. Rev. B 45, 6234 (1992).

${ }^{4}$ D.H. Robertson, D.W. Brenner, and J.W. Mintmire, Phys. Rev. B 45, 12592 (1992).

5 N. Hamada, S. Sawada and A. Oshiyama, Phys. Rev. Lett. 68, 1579 (1992).

${ }^{6}$ J.W. Mintmire, B.I. Dunlap, and C.T. White, Phys. Rev. Lett. 68, 631 (1992).

${ }^{7}$ For a current review of $1 \mathrm{D}$ electrical and thermal conduction in nanowires and nanotubes see, for example, S. Ciraci, A. Buldum, and I. Batra, J. Phys.: Condens. Matter 13, 537 (2001).

${ }^{8}$ Z. Yao, C.L. Kane, and C. Dekker, Phys. Rev. Lett. 84, 4613 (2000).

${ }^{9}$ A. Kleiner and S. Eggert, Phys. Rev. B 63, 073408 (2001).

${ }^{10}$ A. Rochefort, P. Avouris, F. Lesage, and D.R. Salahub, Chem. Phys. Lett. 297, 45 (1998).

${ }^{11}$ A. Rochefort, P. Avouris, F. Lesage, and D.R. Salahub, Phys. Rev. B 60, 13824 (1999).

${ }^{12}$ L. Liu, C.S. Jayanthi, M. Tang, S.Y. Wu, T.W. Tombler, C. Zhou, L. Alexseyev, J. Kong, and H. Dai, Phys. Rev. Lett. 84, 4950 (2000).

${ }^{13}$ T.W. Tombler, C. Zhou, L. Alexseyev, J. Kong, H. Dai, L. Liu, C.S. Jayanthi, M. Tang, and S. Wu, Nature (London) 405, 769 (2000)

${ }^{14}$ S.A. Chesnokov, V.A. Nalimova, A.G. Rinzler, R.E. Smalley, and J.E. Fischer, Phys. Rev. Lett. 82, 343 (1999).

${ }^{15}$ P.E. Lambert, P. Zhang, and V. Crespi, Phys. Rev. Lett. 84, 2453 (2000)

${ }^{16}$ J.P. Salvetat, G.A.D. Briggs, J. Bonard, R.R. Bacsa, A.J. Kulik, T. Stöckli, N.A. Burnham, and L. Forró, Phys. Rev. Lett. 82, 944 (1999).

${ }^{17}$ T. Yildirim, O. Gülseren, Ç. Kılıç, and S. Ciraci, Phys. Rev. B 62, 12648 (2000).
${ }^{18}$ L. Yang, M.P. Anantram, J. Han, and J.P. Lu, Phys. Rev. B 60, 13874 (1999).

${ }^{19}$ L. Yang and J. Han, Phys. Rev. Lett. 85, 154 (2000).

${ }^{20}$ A. Bezryadin, A.R.M. Verschueren, S.J. Tans, and C. Dekker, Phys. Rev. Lett. 80, 4036 (1998).

${ }^{21}$ W. Shen, B. Jiang, B.S. Han, and S. Xie, Phys. Rev. Lett. 84, 3634 (2000).

${ }^{22}$ V. Lordi and N. Yao, J. Chem. Phys. 109, 2509 (1998).

${ }^{23}$ M.F. Yu, T. Kowalewski, and R.S. Ruoff, Phys. Rev. Lett. 85, 1456 (2000).

${ }^{24}$ C.J. Park, Y.H. Kim, and K.J. Chang, Phys. Rev. B 60, 10656 (1999).

${ }^{25}$ M.S.C. Mazzoni and H. Chacham, Appl. Phys. Lett. 76, 1561 (2000).

${ }^{26}$ Ç. Kılıç, S. Ciraci, O. Gülseren, and T. Yildirim, Phys. Rev. B 62, 16345 (2000).

${ }^{27}$ H.S. Sim, C.J. Park, and K.J. Chang, Phys. Rev. B 63, 073402 (2001).

${ }^{28}$ M.C. Payne, M.P. Teter, D.C. Allen, T.A. Arias, and J.D. Joannopoulos, Rev. Mod. Phys. 64, 1045 (1992).

${ }^{29}$ J.P. Perdew and Y. Wang, Phys. Rev. B 46, 6671 (1992).

${ }^{30}$ D. Vanderbilt, Phys. Rev. B 41, 7892 (1990).

${ }^{31}$ G.P. Francis and M.C. Payne, J. Phys.: Condens. Matter 2, 4395 (1990).

${ }^{32}$ H.J. Monkhorst and J.D. Pack, Phys. Rev. B 13, 5188 (1976).

${ }^{33}$ D. Sanchez-Portal, E. Artacho, J.M. Soler, A. Rubio, and P. Ordejon, Phys. Rev. B 59, 12678 (1999).

${ }^{34}$ X. Blase, L.X. Benedict, E.L. Shirley, and S.G. Louie, Phys. Rev. Lett. 72, 1878 (1994).

${ }^{35}$ P. Delaney, H.J. Choi, J. Ihm, S.G. Louie, and M.L. Cohen, Nature (London) 391, 466 (1998).

${ }^{36}$ P. Delaney, H.J. Choi, J. Ihm, S.G. Louie, and M.L. Cohen, Phys. Rev. B 60, 7899 (1999).

${ }^{37}$ O. Gülseren, T. Yildirim, and S. Ciraci, Phys. Rev. Lett. 87, 116802 (2001).

${ }^{38}$ Highlights in Condensed Matter Physics and Future Prospects, edited by L. Esaki, Vol. 285 of NATO Advanced Science Institute (Plenum, New York, 1991).

${ }^{39}$ L. Colombo, R. Resta and S. Baroni, Phys. Rev. B 44, 5572 (1991). 